\title{
Die Ganztagskoordination und ihre Bedeutung für die multiprofessionelle Kooperation an Ganztagsschulen. Eine explorative Fallstudie
}

\author{
Martin Reinert 1
}

Angenommen: 14. April 2020 / Online publiziert: 30. April 2020

(C) Der/die Autor(en) 2020, korrigierte Publikation 2020

Zusammenfassung Neben dem ,Mehr an Zeit' ist die multiprofessionelle Kooperation zu einem wesentlichen Merkmal von Ganztagsschulen geworden. An die Zusammenarbeit zwischen unterschiedlich pädagogisch Tätigen werden Erwartungen gesetzt, da multiprofessionelle Teams gemeinsam erweiterte Bildungsinhalte und Lernerfahrungen für Schüler/innen ermöglichen sollen. Die Ganztagsschule expandiert so zu einem Ort mit erhöhtem Kooperationsbedarf und konfrontiert die schulischen Akteure mit zusätzlichen Anforderungen. Um den Herausforderungen der multiprofessionellen Kooperation nachzukommen, können Schulleiter/innen u. a. auf sog. Ganztagskoordinationen zurückgreifen. Aktuell lässt sich aufgrund des Mangels an einschlägigen Forschungsbefunden jedoch nur spekulieren, wodurch sich die Tätigkeiten von Ganztagskoordinator/innen tatsächlich auszeichnen. Zwar beschreiben einige Studien und Praxisempfehlungen die multiprofessionelle Kooperation als relevanten Ausschnitt des Aufgabenfeldes von Ganztagskoordinator/innen und weisen diesbezüglich auf erforderliche Kompetenzen hin, der konkrete Wirkungszusammenhang der Kooperation bleibt dabei aber unberührt. Ziel dieses Beitrags ist es daher, anhand einer Fallstudie grundlegend der Frage nach der Bedeutung der Ganztagskoordination für die multiprofessionelle Kooperation an Ganztagsschulen nachzugehen. Es zeigt sich, dass die Ganztagskoordination nicht nur eine strukturell wichtige Position in der Ganztagsschule einnimmt, sondern auch, dass sie gezielt für eine Aufrechterhaltung pädagogischer Zuständigkeitsbereiche genutzt wird.

Schlüsselwörter Ganztagsschule · Ganztagskoordination · Multiprofessionelle Kooperation · Fallstudie $\cdot$ StEG-Kooperation

\footnotetext{
M. Reinert, M.A. ( $\bowtie)$

Zentrum für Lehrerbildung (ZfL), Justus-Liebig-Universität Gießen, Bismarckstr. 37, 35390 Gießen, Deutschland

E-Mail: martin.reinert@zfl.uni-giessen.de
} 


\title{
All-day coordination and its significance for multi-professional collaboration in German all-day schools. An exploratory case-study
}

\begin{abstract}
In addition to the 'more of time', multi-professional collaboration has become an essential feature of all-day schools. As multi-professional teams should enable pupils to extend their learning experiences, certain expectations are placed on the collaboration between people with different educational backgrounds. The allday school thus expands into a place with an increased need for collaboration and confronts the different pedagogicals with additional requirements. In order to meet the challenges of multi-professional collaboration, school principals can rely on socalled "all-day coordinators". Due to the lack of relevant research findings, it can only be speculated what actually constitutes the activities of all-day coordinators. Although some studies and practical recommendations describe multi-professional collaboration as a relevant part of the tasks of all-day coordinators and point out necessary competences, the concrete interrelationship of the collaboration remains unaffected. Hence, the aim of this article is to use a case study to fundamentally question the significance of all-day coordinators for multi-professional collaboration at German all-day schools. The article shows that the all-day coordination occupies not only a structurally important position in all-day schools, it is also used to maintain pedagogical areas of responsibility.
\end{abstract}

Keywords All-day schools · All-day coordinators $\cdot$ Multi-professional collaboration $\cdot$ Case-study $\cdot$ StEG-Kooperation

\section{Einleitung}

Aktuell sind Ganztagsschulen in Deutschland mit 69\% aller schulischen Verwaltungseinheiten häufiger anzutreffen als Halbtagsschulen (KMK 2019). Folgt man zudem den Zahlen der letzten Jahre, so belegen diese einen nahezu linearen Ausbautrend von Ganztagsschulen, der voraussichtlich in den nächsten Jahren weiter anhalten wird (Arnoldt et al. 2018). Neben dem ,Mehr an Zeit“ bieten Ganztagsschulen besonders durch die Vielfalt des pädagogischen Personals besonderes Potenzial. In multiprofessionellen Teams sollen die pädagogisch Tätigen gemeinsam u.a. erweiterte Bildungsinhalte für Schüler/innen ermöglichen (Bertelsmann Stiftung 2017). Die Ganztagsschule expandiert so zu einem Ort mit erhöhtem Kooperationsbedarf (Rehm 2018), der schulische Akteure mit zusätzlichen Anforderungen konfrontiert. Denn die Verbesserung der multiprofessionellen Kooperation wird als wesentliches und zu entwickelndes Qualitätsmerkmal von Ganztagsschulen verstanden (Dollinger 2014).

Da mit der multiprofessionellen Kooperation nicht nur erweiterte Anforderungen an die Einzelschule einhergehen, sondern zugleich ein grundlegender Koordinationsaufwand entsteht, können Schulleitungen auf sog. Ganztagskoordinator/innen als zusätzliche Steuerungsinstanz zurückgreifen (vgl. Fischer et al. 2013). So überrascht es nicht, dass neben den organisatorischen Aufgaben für den pädagogischen Alltag an Ganztagsschulen auch die Kooperation mit den verschiedenen pädagogisch Täti- 
gen als relevante Zielgröße in der Arbeit von Ganztagskoordinator/innen aufgeführt wird. Hierunter können bspw. Aufgaben wie die Suche nach Gemeinsamkeiten in der Kooperation, besonders aber auch das Erkennen und Verdeutlichen von Grenzen fallen. Aufgrund des Mangels an einschlägigen Forschungsbefunden lässt sich aktuell jedoch nur spekulieren, wodurch sich die Tätigkeiten von Ganztagskoordinator/innen tatsächlich auszeichnen. So konzentrieren sich die Forschungsergebnisse auf eine Auflistung bestehender Kooperationspartner/innen und berufen sich nahezu ausschließlich auf Selbstbeschreibungen eingesetzter Ganztagskoordinator/innen. Das Ergebnis sind Praxis- und Handlungsanweisungen, in denen die Perspektive des gesamten Personals nicht berücksichtigt und damit auch die Kooperation in ihrem Wirkungszusammenhang nicht ersichtlich wird. Ziel des vorliegenden Beitrags ist es daher, anhand einer Fallstudie und unter Nutzung der im Rahmen der Gießener Teilstudie der Studie zur Entwicklung von Ganztagsschulen (StEG) entwickelten Netzwerkkarten und Leitfadeninterviews einer offenen Ganztagsschule tiefergehend nach der Bedeutung von Ganztagskoordinator/innen für die multiprofessionelle Kooperation an Ganztagsschulen zu fragen.

\section{Forschungsstand}

\subsection{Multiprofessionelle Kooperation}

Die heterogene Zusammensetzung des pädagogischen Personals ist zu einem wesentlichen Merkmal von Ganztagsschulen geworden (Coelen und Rother 2014). So geben in einer aktuellen Schulleitungsbefragung etwa neun von zehn Ganztagsschulleitungen an, neben Lehrer/innen mit weiterem pädagogisch tätigem Personal zu arbeiten (StEG-Konsortium 2019). Die Ganztagsschule wird so zu einem Ort, an dem Lehrkräfte mit einer großen Bandbreite an weiterem Personal zusammenarbeiten (Kielblock und Gaiser 2017). Kooperieren mehr als zwei Berufsgruppen an einem Ort miteinander, die einen gewissen Grad an Spezialisierung aufweisen, koordinieren diese ihre Handlungen und tauschen gegenseitig, u. U. auch unter Beibehaltung von Hierarchien, Expertise aus, dann spricht man von multiprofessioneller Kooperation (vgl. Speck et al. 2011). In multiprofessionellen Teams soll die Vielfalt des Personals zielführend eingesetzt werden, um bspw. gemeinsam erweiterte Bildungsinhalte für Schüler/innen zu ermöglichen (Bertelsmann Stiftung 2017).

Im Entstehungszeitraum des vorliegenden Beitrages fällt auf, dass der Forschungsstand zur multiprofessionellen Kooperation an Ganztagsschulen wesentlich umfangreicher ausfällt als die Forschungsbefunde zum konkreten Tätigkeitsfeld der Ganztagskoordination. So finden sich Befunde zur Praxis der Zusammenarbeit zwischen den pädagogisch Tätigen, die auf Differenzierungspraktiken bei der Bearbeitung pädagogischer Probleme verweisen. Reh und Breuer (2012, S. 196) arbeiten u.a. heraus, dass situationsbezogene Adressierungen ,entlang professionsspezifischer Zuständigkeiten“ eine Zusammenarbeit der beteiligten Professionen auf Augenhöhe ermöglichen, jedoch unter Aufrechterhaltung ihrer fachlichen Differenzierung. Dass Positionierungen gegenüber Zuständigkeiten aufgrund fehlender Einblicke in Tätigkeitsbereiche auch vermieden werden, zeigen die Ergebnisse 
von Silkenbeumer et al. (2018). Den Autor/innen zufolge kann der mangelnde Einblick in das gegenseitige Tätigkeitsfeld dazu führen, dass Kooperation nicht als organisatorisches Problem, sondern als Beziehungsproblem ausgehandelt wird. Die Zuweisung bestimmter Aufgaben und Teilverantwortungen muss jedoch nicht der positiven Sicht der Beteiligten auf Kooperation widersprechen, sondern kann im gegenseitigen Einvernehmen etabliert sein (Buchna et al. 2016).

Auch das Verhältnis zwischen den pädagogisch Tätigen wird zum Ausgangspunkt unterschiedlicher Forschungsarbeiten. Gröhlich et al. (2015) kommen zu dem Ergebnis, dass aus Sicht der Lehrer/innen von Ganztagsgymnasien eine Kultur der gegenseitigen Wertschätzung besteht. Eine ähnlich positive Einschätzung findet sich auch aufseiten des weiteren pädagogischen Personals. Dass sich die Kooperationsbeziehungen auch anders darstellen können, zeigen Olk et al. (2011). Zwar weisen die Autor/innen auf professionsübergreifende Partizipationsmöglichkeiten hin, ein Verzicht auf die dominierende Perspektive von Lehrer/innen lässt sich in der Zusammenarbeit jedoch nicht allgemein beobachten, weshalb eher von einem Nebenund nicht von einem Miteinander zwischen den pädagogischen Akteuren gesprochen werden kann.

Bereits dieser „,kleine“ Überblick soll aufzeigen, dass es sich bei der multiprofessionellen Kooperation um einen facettenreichen und viel beforschten Gegenstand handelt, der von wesentlichen Hoffnungen an eine gelingende Kooperation begleitet wird (Rahm et al. 2015). Das ,Gelingen“" ist dabei an Stellschrauben gebunden, die die Einzelschulen aufgrund der zögerlichen Implementierung neuer Kooperationsstrukturen tagtäglich herausfordern (de Boer und Spies 2014). Dennoch wird die Verbesserung der multiprofessionellen Kooperation als eine der zentralen Aufgaben der Entwicklung von Ganztagsschulen angesehen (Dollinger 2014). Insofern ist es nicht verwunderlich, dass im Zuge der Ganztagsschulentwicklung durch die Implementierung sog. Ganztagskoordinator/innen eine Erweiterung des Handlungsspielraumes der Einzelschulen angestrebt wurde.

\subsection{Ganztagskoordinator/innen}

Die Implementierung von Ganztagskoordinator/innen wird auf die erweiterten Handlungsanforderungen zurückgeführt, mit denen sich Schulen im Zuge der Einführung des Ganztags konfrontiert fühlen. Zwar liegen die mit der Durchführung von Ganztagsangeboten einhergehende Organisation und Aufsicht der veränderten strukturellen, personellen und zielbezogenen Anforderungen im Verantwortungsbereich der Schulleitung. Sie kann jedoch mithilfe der Ganztagskoordination auf eine zusätzliche Steuerungsinstanz zurückgreifen (Fischer et al. 2013). Aktuell liegen wenige Forschungsbefunde über das konkrete Tätigkeitsprofil von Ganztagskoordinator/innen vor. Zwar finden sich repräsentative Studien über die Zusammensetzung von Steuerungsgremien an Ganztagsschulen (StEG-Konsortium 2017)1, deren Wahrnehmung seitens der pädagogisch Tätigen (Steiner und Tillmann 2011) sowie Beschreibungen über Koordinationsmodelle in NRW (Kahnert et al. 2015). Das durch die Studien

\footnotetext{
1 U.a. die Schulleitung, Personen aus dem Lehrerkollegium, Personen aus dem pädagogischen Personal oder eine Steuergruppe (StEG-Konsortium 2017, S. 187).
} 
hervorgebrachte Beschreibungswissen gibt jedoch nur bedingt Einblicke in das Tätigkeitsfeld von Ganztagskoordinator/innen. Es ließen sich nur wenige Publikationen finden, aus denen konkrete Einblicke in das Tätigkeitsfeld von Ganztagskoordinator/innen hervorgehen. $\mathrm{Zu}$ nennen wären hier zum einen die von der Bertelsmann Stiftung (2017) veröffentlichten „Empfehlungen für einen guten Ganztag“, in denen festgehalten wird, dass die organisatorischen Aufgaben für den pädagogischen Alltag an Ganztagsschulen von einer Ganztagskoordination übernommen werden können. Zum anderen arbeiten Kahnert et al. (2015) relevante Handlungsfelder und erforderliche Kompetenzen für Ganztagskoordinator/innen heraus und stellen diese in Form von Praxistipps zur Verfügung. Schließlich wird das umfangreiche Aufgabenfeld von Ganztagskoordinator/innen von Altermann et al. (2018) als Begründung für erste Qualitätsstandards an die damit verbundene Stelle aufgeführt. So gilt es, dem gestiegenen Anforderungsprofil von Ganztagskoordinator/innen durch Mindestqualifikationen gerecht zu werden.

Mit Blick auf die multiprofessionelle Kooperation zeigt sich, dass sich die Notwendigkeit zur Kooperation zwar in allen referierten Forschungsarbeiten für Ganztagskoordinator/innen findet, d.h. sie wird auch von allen Stelleninhaber/innen erwähnt, diese Thematisierung gleicht jedoch einer Aufsummierung relevanter Kooperationspartner und einer (Selbst-)Zuschreibung notwendiger Kompetenzen. Die Forschungsergebnisse verweisen insofern nur darauf, dass es sich bei der multiprofessionellen Kooperation um einen relevanten Ausschnitt des Aufgabenfeldes von Ganztagskoordinator/innen handelt, für den spezifische Kompetenzen erforderlich sind. Es wird hingegen nicht klar, in welchem Wirkungszusammenhang die Aufgaben und Kompetenzen in den Schulen stehen, da aktuell keine tiefergehenden Fragestellungen und Analysen darüber existieren.

\subsection{Ganztagskoordinator/innen und Grenzziehungen}

Dass Ganztagskoordinator/innen eine tragende Rolle in der multiprofessionellen Kooperation spielen können, wird ersichtlich, wenn Kooperation als grundlegendes Spannungsverhältnis verstanden wird, in dem pädagogische Akteure Grenzziehungen nutzen, um Zuständigkeiten aushandeln und dauerhaft bewältigen zu können (Bauer 2014). Grenzziehungen sind in diesem Sinne „Differenzleistungen“ (Weidenhaus 2015, S. 46) zwischen zwei oder mehreren (pädagogischen) Bereichen, für die die Interaktionen der Akteure eine wesentliche Rolle spielen (Akkerman und Bakker 2011). Als Verständnishilfe bieten sich basale Fragestellungen zur Organisation der Ganztagsschule an: Wo hört beispielsweise der Zuständigkeitsbereich der Schule auf? Wo fängt dagegen die Zuständigkeit der Nachmittagsbetreuung an? Zielen derartige Fragen eher auf potenzielle Auflösungserscheinungen unhinterfragt geltender Grenzen in der Ganztagsschule ab, so rücken sie zugleich die damit einhergehende Grenzarbeit der Akteure in den Fokus (Rahm et al. 2015). Denn Ganztagsschulen lassen sich als Orte verstehen, an denen professionelle Identitäten aufgrund erweiterter Kooperationsanforderungen in Frage gestellt werden.

Grenzziehungen werden von Bauer (2014) zum Anlass genommen, um neben den strukturellen Bedingungen der Schule besonders die Strategien der Akteure zur Aufrechterhaltung von Handlungsspielräumen in den Fokus zu rücken. Zudem weist 
sie darauf hin, dass auch Positionierungspraktiken (vgl. Abschn. 2.1) als ganztagsspezifische Grenzziehungsprozesse gedacht werden können. Unberücksichtigt bleibt bisher die Frage, ob durch die Implementierung von Ganztagskoordinator/innen das strategische Arsenal für Grenzziehungsprozesse erweitert wurde. Schließlich handelt es sich bei der professionsübergreifenden Kooperation um eine Tätigkeit von Koordinator/innen, über die aktuell keine tiefgründigen Analysen vorliegen. Ein Forschungsdesiderat stellt somit die Frage dar, ob durch die Arbeit von Ganztagskoordinator/innen professionsübergreifende Kooperationsmöglichkeiten geschaffen oder diese von den Akteuren gezielt für die Aufrechterhaltung getrennt agierender Bereiche genutzt werden.

\section{Fragestellung}

Der vorliegende Beitrag nimmt das aufgeworfene Forschungsdesiderat zum Ausgangspunkt und zielt darauf ab, die Bedeutung der Ganztagskoordination für die multiprofessionelle Kooperation an Ganztagsschulen aus der Perspektive des pädagogischen Personals zu analysieren. Es geht demnach nicht um Selbstbeschreibungen, sondern um die Fremdzuschreibung relevanter Aspekte für die Kooperation. Diesem Ansatz folgend wird das Tätigkeitsfeld der Ganztagskoordination in ihrem einzelschulischen Wirkungszusammenhang betrachtet. Im Zentrum stehen dabei folgende Fragestellungen:

\section{Kann die Ganztagskoordination als Grenze zwischen den Professionen identifiziert werden?}

Zunächst wird explorativ analysiert, wie sich die auf den Netzwerkkarten befindlichen Positionen der Ganztagskoordination zu den Positionen der jeweils anderen Profession interpretieren lassen.

\section{Wie zeichnet sich die Grenzarbeit der Ganztagskoordination aus?}

Sollte das erste Analyseergebnis die Ganztagskoordination als Grenze identifizieren, so soll in der weiterführenden Analyse des Interviewmaterials deduktiv geklärt werden, ob mit dieser Verortung eine spezifische Grenzarbeit verbunden ist.

3. Welche Bedeutung hat die Ganztagskoordination für die multiprofessionelle Kooperation?

Hierbei handelt es sich um die eigentliche Leitfrage des Beitrags. Aufgrund fehlender Forschungsbefunde wurde diese bewusst explorativ formuliert. Zudem sollte die offene Fragestellung für größtmögliche Irritation für die potenziell deduktiv berücksichtigte Grenzkategorie sorgen.

\section{Methode}

Für die Beantwortung der Leitfragen wird den „Stimmen aus der Praxis“ durch die Nutzung einer qualitativen Fallstudie besondere Aufmerksamkeit geschenkt (Flick 
et al. 2017). Die Bedeutung von Ganztagskoordinator/innen für multiprofessionelle Kooperation kann dadurch am Schulstandort nicht nur detailreich analysiert, sondern auch in ihrer Charakteristik umfangreich beschrieben werden, ohne konstituierende Rahmenbedingungen zu vernachlässigen. Letztere erscheinen als wesentliche Faktoren, um den Wirkungszusammenhang der multiprofessionellen Kooperation am Beispiel einer Ganztagsgrundschule (im Folgenden Sonnenschule ${ }^{2}$ genannt) verstehen zu können. Durch die akribische Betrachtung eines Falles können nicht nur ein gegenstandsnahes Tiefenverständnis, sondern auch Folgehypothesen resultieren (Johnson und Christensen 2017).

\subsection{Datenerhebung}

Die Datenerhebungen fanden im Rahmen des Gießener Teilprojekts der BMBFgeförderten Studie zur Entwicklung von Ganztagsschulen (StEG; 2016-2019) statt. Insgesamt wurden 28 Mitarbeiter/innen per Leitfadeninterviews über die Bedeutung und die konkrete Ausgestaltung der multiprofessionellen Kooperation am Schulstandort befragt. Zudem wurden die Befragten darum gebeten, ihre alltägliche Zusammenarbeit in einer Netzwerkkarte festzuhalten (vgl. Abb. 1). Im Zentrum des Interesses stand dabei, welche Personen von den Befragten in den konzentrischen Kreisen besonders nah (intensive Kooperation) oder fern (weniger intensive Kooperation) verortet werden. So konnten mehrere Kooperationspartner/innen auf unterschiedlichen maßstäblichen Ebenen abgebildet und in Relation gesetzt werden, die sich um die Befragten aufbauten. Zusätzlich wurden die Befragten darum gebeten, während ihrer Eintragungen laut zu denken. Im Analyseprozess konnte so nachvollzogen werden, welche Gründe zu den Verortungen führten. Ergänzend wurden einmalig durchgeführte teilnehmende Beobachtungen genutzt, um ein Bild über die Kooperationszusammenhänge am Standort zu bekommen.

\subsection{Datenanalyse}

Die Datenanalyse setzte sich aus zwei aufeinander bezogenen Analyseschritten zusammen. In einem ersten Schritt sollte anhand der Netzwerkkarten die erste Leitfrage des Beitrags - Ganztagskoordinator/innen als Grenze - überprüft werden. Im Zentrum der Analyse stand der explorative Vergleich zwischen den Eintragungen der Lehrkräfte mit denen des weiteren pädagogischen Personals. Hierfür wurden die Netzwerkkarten professionshomogen zusammengefasst. Aufgrund der vielfältigen Eintragungen erschien es notwendig, klare Unterscheidungen zwischen den Akteuren und Institutionen zu verwenden. So kam es dazu, in den Netzwerkkarten der Lehrkräfte einerseits die Verortung der Ganztagskoordination, andererseits nur jene Eintragungen als Vergleichsgröße zuzulassen, aus denen der klare Verweis auf die kooperierende Institution hervorging. Bei den Netzwerkkarten der weiteren pädagogisch Tätigen wurden dagegen die Ganztagskoordination sowie der klare Bezug zur Schule berücksichtigt. Abschließend erfolgten die Zusammenführung und der Vergleich der Gesamtergebnisse (vgl. Abschn. 6.1; Abb. 1).

\footnotetext{
2 Alle Namen und Orte wurden anonymisiert.
} 
Für die Analyse der Leitfadeninterviews wurde die qualitative Inhaltsanalyse nach Mayring (2015) gewählt, da durch diese sowohl der explorative Charakter der Studie als auch die deduktive Berücksichtigung des ersten Analyseergebnisses abgebildet werden können. Um das Ergebnis der Netzwerkanalyse berücksichtigen zu können, wurde die Untersuchung zum einen durch das „Boundary Work“ Konzept nach Allen (2000) sowie durch Beiträge, die sich dem Handlungsfeld sog. „Grenzgänger“ (u. a. broker) widmen (vgl. hierzu Akkerman und Bakker 2011), angereichert. Das Datenmaterial konnte so nach rhetorischen Grenzziehungsstrategien zwischen den Professionen untersucht werden. Die induktive Kategorienbildung wurde dagegen als zielführender Ansatz gewählt, da es aufgrund des skizzierten Forschungsdesiderats wichtig war, das Material gegenstandsnah und möglichst theoriefrei zu analysieren (Mayring 2015).

\section{Vorstellung der Sonnenschule}

Die Sonnenschule liegt in Bergstadt, ist seit zwei Jahren offene Ganztagsschule und stellt die Betreuung von ca. 170 Schüler/innen durch drei pädagogische Bereiche sicher. So finden sich vor Ort die Schule, der ,schulische Ganztag', der unter der Verantwortung der Schulleitung von einer Ganztagskoordinatorin („Frau Huber") geleitet wird, sowie zwei kooperierende Horte. Der ,schulische Ganztag " und Hort 1 befinden sich mit ihren Betreuungsangeboten in einem gemeinsamen Gebäude. Hort 2 ist dagegen separat untergebracht. Durch die Verteilung des Ganztags auf verschiedene Bereiche sprechen die pädagogisch Tätigen von verlängerten Wegen und einem erschwerten Austausch. Zum Zeitpunkt der Feldphase wird von keiner „festgelegte[n] Kooperation“ zwischen den beteiligten Institutionen gesprochen, weshalb auch das Anmeldeverfahren für spezifische Angebote im Ganztag oder in den Horten als Herausforderung im Schulalltag hervortritt. Es zeigen sich gewachsene Strukturen, die von getrennten Bereichen ausgehen und bei den Hortmitarbeitenden teilweise das Gefühl hervorrufen, bei Entscheidungsfindungen außen vor zu sein. Am Schulstandort wird Zeit immer wieder als wichtige Voraussetzung für Kooperation genannt, aufgrund der Aufteilung auf die genannten Institutionen aber auch sehr häufig bemängelt. So verlassen die Lehrkräfte die Schule, bevor die Ganztags- oder Hortmitarbeitenden zu arbeiten beginnen. Dies führt dazu, dass sich die Kooperation auf wöchentlich stattfindende und professionshomogene Teamtreffen konzentriert, um den pädagogischen Alltag in den jeweiligen Zuständigkeitsbereichen zu planen. Ein konkretes Aufeinandertreffen der einzelnen Bereiche findet ausschließlich im institutionalisierten Leitungstreffen statt, an dem die Leitungen der Horte, die Schulleitung und die Koordinatorin teilnehmen. Dort getroffene Entscheidungen und ausgetauschte Informationen fließen anschließend über die Leitungen in deren Zuständigkeitsbereiche. Fraglich bleibt jedoch, ob die Informationen verlässlich die Mitarbeitenden erreichen. In Bezug auf ihren pädagogischen Alltag werden von den Beteiligten daher nur punktuelle Überschneidungen genannt, denen in der Regel ein konkreter Kooperationsanlass zugrunde liegt und die sich auf Tür-undAngel-Gespräche konzentrieren. Aufgrund des räumlichen und zeitlichen Mangels an Überschneidungen kommt es zu einer Konzentration der Kooperation auf die 
Ganztagskoordinatorin. Dies führt zu einer gegenseitigen Adressierung in Not- und Ausnahmefällen sowie einem ,Nicht-Sehen ' und,Nicht-Kennen ' der Akteure. Zudem lässt sich festhalten, dass die Ganztagskoordination förmlich mit Aufgaben überhäuft wird. Eine derartige Überfrachtung von Tätigkeiten belastet dabei nicht nur die Koordinatorin selbst, sondern wird auch zur Belastungsprobe für den Schulstandort. So spricht die Schulleitung von einer „Katastrophe“, sollte die Ganztagskoordinatorin ausfallen.

\section{Ergebnisse}

In der Ergebnisdarstellung wird eine Trennung zwischen dem Ergebnis der Netzwerkanalyse und dem Ergebnis der Leitfadeninterviews vorgenommen, da es sich bei der Antwort auf die Frage, ob die Ganztagskoordination eine Grenze für die Kooperation zwischen den Professionen ist, um eine relevante Perspektive auf die weiterführenden Analysen handelt.

\subsection{Ganztagskoordination als Grenze zwischen den Professionen}

Die Zusammenführung der Netzwerkkarten der Lehrkräfte brachte Abb. 1 hervor. Bei den dunklen Punkten handelt es sich um die von den Lehrkräften festgehaltenen Positionen der Ganztagskoordination, die weißen Punkte stellen dagegen die Positionen des weiteren pädagogischen Personals dar. Die quadratische Trennlinie soll an dieser Stelle als Verständnishilfe dienen, um die (räumlichen) Relationen der Markierungen und damit auch die Annahme einer Grenze zu erleichtern. Mithilfe der Trennlinie fällt auf, dass sich die intensive Kooperation (näher am Zentrum) primär auf die Ganztagskoordinatorin konzentriert. Zwar scheinen intensive Kooperationsprozesse auch punktuell mit Mitarbeiter/innen der jeweils anderen Profession stattzufinden, diese zeigen sich jedoch nur in geringem Maße. So befindet sich die Mehrzahl des weiteren pädagogischen Personals im letzten konzentrischen Kreis, was für eine niedrigere Kooperationsintensität spricht. Wie auch in der Fallstudie beschrieben, verläuft die Kooperation am Standort über die Ganztagskoordinatorin und konzentriert sich demnach auf Verfahrens- und Organisationsabläufe. Thematisch wären hier Gestaltungsaufgaben verbunden mit Teamsitzungen oder Steuerungstreffen vorstellbar (Maykus 2009). Untermauert man die Interpretation mit einer für den Kooperationskontext typischen Verbesserungswürdigkeit räumlicher und zeitlicher Überschneidungen (bspw. bei Chiapparini et al. 2018), so lässt sich festhalten, dass ein ,gelebtes Miteinander' zwischen den pädagogisch Tätigen nicht zu finden ist. Die Kooperation würde sich stets auf einen professionshomogenen Personenkreis und die Ganztagskoordination konzentrieren. Die mit dem Ganztag erhofften und zum Teil normativ aufgeladenen Synergiebestrebungen zwischen den Professionen würden in dieser Lesart ausbleiben, da sich die Ganztagskoordinatorin in den Netzwerkkarten überwiegend als Punkt der maximalen Ausweitung der Kooperation darstellt. Oder kurz: Am Schulstandort ist sie jener Punkt zwischen den Professionen, an dem die Kooperationsprozesse enden. Für die Frage, ob es sich bei der Ganztagskoordination um eine Grenze für die Kooperation zwischen den Professionen handelt, ist gerade 


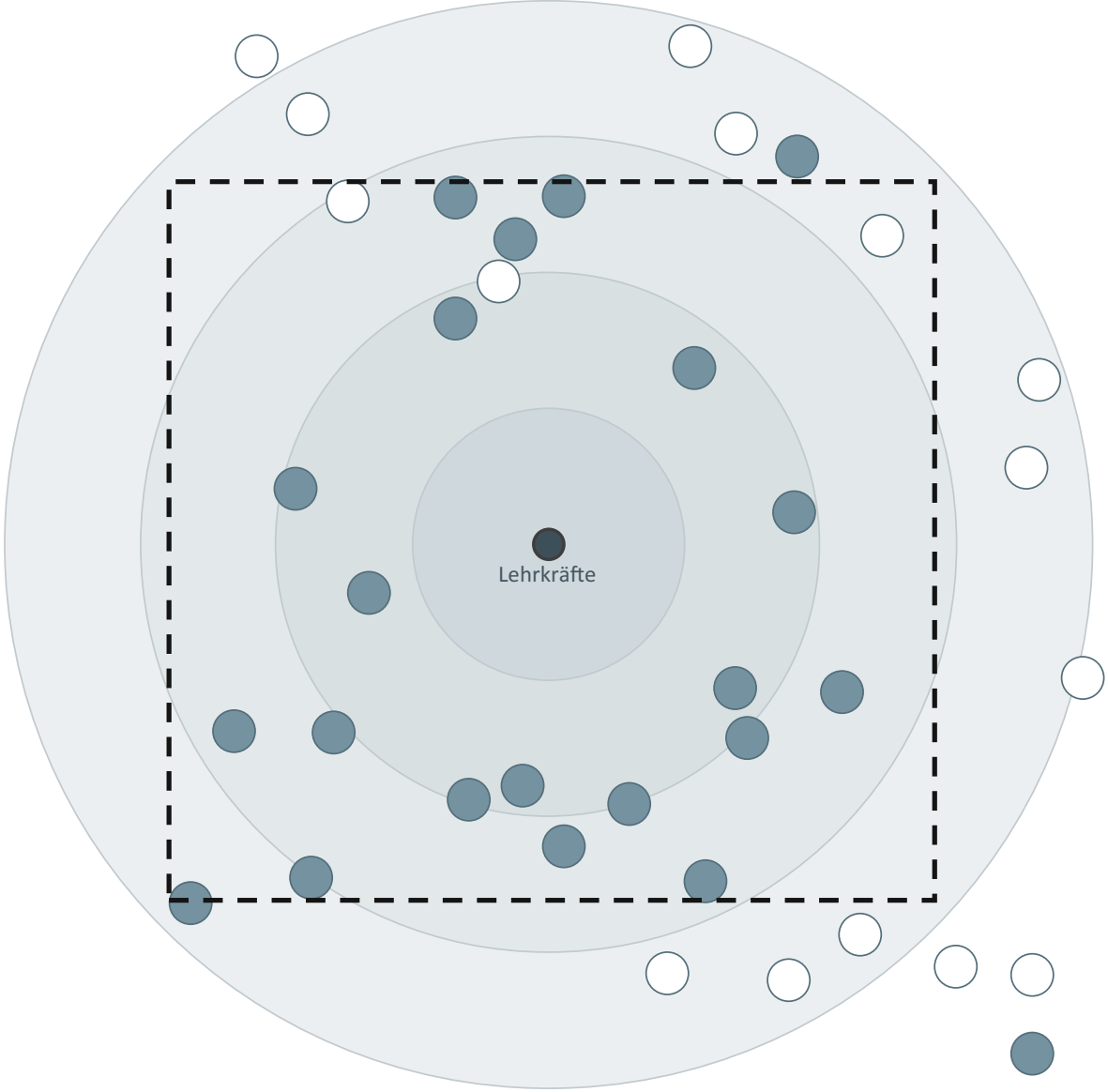

Abb. 1 Zusammenführung der Verortungen der Lehrkräfte

die maximale Ausweitung der Kooperationsprozesse besonders wichtig, da Grenzen in der Zusammenarbeit durch soziokulturelle Differenzen entstehen können, die zu Abbrüchen in der Kommunikation und Interaktion führen (Akkerman und Bakker 2011). In diesem Sinne kann auch die Ganztagskoordination als Grenze verstanden werden.

\subsection{Die Grenzarbeit der Ganztagskoordination}

Den Ergebnissen der Netzwerkanalyse folgend, spielt sich die Kooperation zwischen den Professionen am Standort über die Ganztagskoordination und somit im Rahmen des ,Sich-nicht-Sehens' bzw. ,Sich-nicht-Kennens' ab. Dies spiegelt sich auch in den Aussagen der pädagogisch Tätigen wider. Es finden sich Aussagen wie die folgende: „Frau Huber [...] mit der habe ich sehr viel Kontakt, [...] aber mit den Lehrern, wenn sie darauf anspielen, habe ich überhaupt keinen Kontakt". Auch das zwischenmenschliche Verhältnis innerhalb dieser Konstellation wird von den 
Akteuren beschrieben. Ein Erzieher hält fest, dass die Kooperation zu den weiteren Professionen am Standort „ein bisschen kompliziert“ sei. Das „getrennte System“ am Schulstandort wird durch die Grenzarbeit der Ganztagskoordinatorin jedoch als vernetzt erlebt. So ist es nicht verwunderlich, dass mehrere Mitarbeiter/innen die Ganztagskoordinatorin als Schlüsselperson einschätzen und festhalten, dass ,diese ganzen [Informations-]Zusammenläufe" nur durch die Arbeit der Koordinatorin funktionieren.

Weiterhin weisen mehrere Aussagen darauf hin, dass die Grenzarbeit zu positiv und zu negativ konnotierten Grenzverwischungen führen kann. Grenzverwischungen können als potenzielle, durch die Grenzarbeit hervorgehende Wirkungen auf die Kooperation verstanden werden. So müssten einer Sozialarbeiterin zufolge nicht nur die Koordinatorin, sondern alle „wissen, was im Ganztag abgeht“. Eine Lehrerin hält ebenfalls fest, dass die anderen Professionen ,,ja eigentlich in dem Sinne auch dazu“ gehören. Den pädagogisch Tätigen fällt durch die intensive Einbindung der Koordinatorin auf, wer an der Kooperation am Standort nicht teilnimmt, es aber im Sinne gemeinsamer Kooperationsprozesse tun müsste. Das gleiche Muster kann jedoch auch verkehrt werden, indem die umfassende Einbindung der Koordinatorin die anderen Bereiche obsolet werden lässt. Das dahinterliegende ,große Ganze“ wird in dieser Argumentation auf die Ganztagskoordination reduziert, indem sie die abwesenden Bereiche verkörpert. Daran geknüpfte Aussagen können sich auf einzelne Bereiche beziehen - ,der Hort 1 ist bei Frau Huber ja in gewisser Weise mit drin“ - oder aber auch bereichsübergreifend sein - ,also Hort fällt bei mir ein stückweit unter Ganztag“.

Schließlich wird die Zusammenarbeit mit der Ganztagskoordinatorin von einzelnen pädagogisch Tätigen für eine Sicherung der Arbeitsgrenzen genutzt. So weist eine Lehrerin auf die funktionierende Kooperation am Schulstandort hin und betont, dass man „die Sachen nebeneinander bestehen lassen“ könne. Das Funktionieren der Zusammenarbeit scheint den Aussagen der Lehrkraft zufolge aber wesentlich auf der Koordinatorin zu fußen, denn bezüglich der bemängelten Abwesenheit der Horte sagt sie: „Aber Frau Huber schafft das auch.“ Ähnlich argumentiert eine weitere Lehrkraft. Dabei wird erneut die Kombination aus hortseitiger ,Nicht-Funktion“ der Zusammenarbeit einer, funktionierenden“ Kooperation mit Frau Huber gegenübergestellt und mit der Aussage resümiert: „Mit Frau Huber klappt das [die Kooperation] schon gut." Festzuhalten bleibt, dass sich das organisatorische Nebeneinander durch die Grenzarbeit der Koordinatorin als ein Miteinander zu erkennen gibt (,Also ich empfinde das schon als Nebeneinander und Miteinander.").

\section{Diskussion}

Der vorliegende Beitrag nahm das Forschungsdesiderat über das Tätigkeitsfeld von Ganztagskoordinator/innen als Ausgangspunkt, um sich anhand einer Fallstudie grundlegend mit der Frage nach der Bedeutung der Ganztagskoordination für die multiprofessionelle Kooperation an Ganztagsschulen zu befassen. Die Analyse setzte sich aus drei aufeinander aufbauenden Leitfragen zusammen. Zunächst wurden Netzwerkkarten analysiert, in denen u.a. Lehrkräfte die Ganztagskoordination als 
Punkt der maximalen Ausweitung der Kooperation aufführten. Die Ganztagskoordination fungiert damit als Grenze für die Zusammenarbeit zwischen den Professionen (vgl. Abschn. 6.1). Das Ergebnis der Netzwerkanalyse wurde weiterführend in die Untersuchung des Interviewmaterials einbezogen, um herauszufinden, welche Grenzarbeiten mit der Position der Ganztagskoordination verbunden sind.

Die Ergebnisse zeigen, dass sich die Bedeutung der Ganztagskoordination für die multiprofessionelle Kooperation an der analysierten Ganztagsschule durch ihre Funktion als Grenzgänger/in begründet. Sie erleichtert die Interaktionen und den Informationsfluss zwischen den pädagogisch Tätigen und nimmt damit eine strukturell wichtige Position ein. Die Ganztagskoordinatorin befindet sich stets an den Grenzbereichen der pädagogischen Berufskulturen und weist damit „das Wissen und den Einblick des Eingeweihten mit der kritischen Attitüde des Außenstehenden“ (Lindner 2007, S. 204) auf. Nicht ohne Grund wird sie von allen Akteuren als zentrale Ansprechpartnerin, bspw. aber auch als ,,irgendwie zum Kollegium“ der Lehrkräfte zugehörig (Eingeweihte) oder die Horte, verkörpernd" und damit als Außenstehende beschrieben. Eine Positionierung wie diese ist prädestiniert für die (Sonder-)Rolle eines Vermittlers/einer Vermittlerin zwischen den Professionen, da diese(r) nicht nur die professionsspezifischen Geheimnisse erfährt, sondern auch Zugang zu allen Regionen des Ganztagsschulstandorts hat (Goffman 2016). Den pädagogisch Tätigen steht mit der Ganztagskoordination ein Übersetzungsmedium zur Verfügung, das ganztagsrelevante Informationen ganzheitlich zu verarbeiten weiß und jede Profession extern zu repräsentieren vermag (vgl. Sendzik et al. 2012).

Zugleich führt der umfangreiche Einbezug der Ganztagskoordinatorin in die multiprofessionelle Kooperation zu Grenzverwischungen. Einerseits dient sie den pädagogisch Tätigen als reflexiver Ausgangspunkt. In diesem Sinne verweist sie auf ausbleibende Kooperationserfordernisse, die jedoch für eine Stärkung der multiprofessionellen Kooperation am Ganztagsschulstandort wünschenswert wären. Andererseits wird die Kooperation mit der Koordinatorin für einzelne Akteure nicht nur als gewinnbringend, sondern als ausreichend empfunden, sodass damit auch das ,große Ganze' der Kooperation verschwimmt. Die anderen Kooperationspartner werden in diesem Sinne für die beteiligten Professionen nach und nach obsolet. Schließlich nutzen einige Akteure die Erfahrung aus der Kooperation mit der Ganztagskoordinatorin, um die Grenzen zwischen den jeweiligen Bereichen zu sichern. Für derartig drastische Abgrenzungen steht das Funktionieren der Kooperation im Vordergrund. So wird bspw. von den Lehrkräften ein Vergleich zwischen der Kooperation mit dem Hort und der Kooperation mit der Ganztagskoordination vorgenommen und als Begründung für den Erhalt getrennter Zuständigkeitsbereiche genutzt.

Auch die vorliegenden Ergebnisse verweisen auf Aushandlungsprozesse zur Aufrechterhaltung pädagogischer Zuständigkeitsbereiche in der Ganztagsschule (hierzu u. a. Reh und Breuer 2012). Dass diese jedoch in einer vereinfachten Koordination zwischen den Beteiligten münden, lässt sich dagegen nicht bestätigen (vgl. hierzu Bauer 2014). Zwar ist die Ganztagskoordination primär für Steuerungs- und Koordinationsaufgaben eingesetzt, dennoch führt die Zentralisierung der Kooperation auf diese Position und damit einhergehend auch die beschriebene Grenzarbeit, zu organisatorischen Herausforderungen. Durch die umfangreiche Einbindung der Koordinatorin werden die Partizipationsmöglichkeiten der pädagogisch Tätigen 
eingeschränkt, sodass das pädagogische Personal als ,,vorbereitendes, aber nicht beschlussfähiges Gremium“ (Breuer und Steinwand 2015, S. 270) erscheint. Mitbestimmungsmöglichkeiten sind jedoch wesentliche Voraussetzungen, damit die Intensität der Kooperation zwischen den Akteuren beflügelt werden kann (Tillmann und Rollett 2011). Der systematische Ausschluss von Kooperationsprozessen führt besonders bei Vertrauensfragen dazu, dass sich die Beteiligten gegenseitig suchen und bspw. über Anwesenheiten von Schüler/innen absichern lassen müssen. Am Schulstandort kann daher von einer ,Zuspitzung der Vertrauensproblematik“ (Fabel-Lamla 2012, S. 202) in der multiprofessionellen Kooperation gesprochen werden, da die Herausbildung einer wechselseitigen Vertrauensbeziehung durch die Ganztagskoordination herausgefordert wird. Dem pädagogischen Personal jedoch Unmündigkeit zuzuschreiben, wäre zu kurz gegriffen. Entgegen der beschriebenen Differenzierungspraktiken (vgl. Abschn. 2.1), ermöglicht der strategische Rückgriff auf die Ganztagskoordination eine , unauffälligere ' Aushandlung von Zuständigkeiten. Unauffällig, da sich das organisatorische Nebeneinander als empfundenes Miteinander zu erkennen gibt. So wird die Ganztagskoordination als Vermittler/in genutzt, damit die pädagogischen Akteure in ihren spezifischen Zuständigkeitsbereichen verbleiben und eine gegenseitige Sichtbarkeit vermeiden können. Dabei ist sie zugleich ein/e Repräsentant/in der beteiligten Professionen. In diesem Sinne lässt sie sich als Schutz der Zuständigkeitsbereiche vor unerwünschtem Zugriff (ähnlich einer Firewall) verstehen (vgl. auch Breuer und Steinwand 2015). Gerade im Zuge zentraler Steuerungselemente (bspw. Systemmonitoring), in denen u. a. die multiprofessionelle Kooperation als Qualitätsmerkmal berücksichtigt wird, bekommt der strategische Rückgriff auf die Koordination besondere Bedeutung. Sie ermöglicht den pädagogisch Tätigen eine strategische ,Pseudokooperation'. Ganz im Sinne: Wir tun das gleiche wie vorher, geben jedoch vor, es nicht zu tun.

Schließlich bleibt zu konstatieren, dass die multiprofessionelle Kooperation Ganztagskoordinator/innen mit Anforderungen und Belastungen konfrontiert, die in den referierten Forschungsbefunden nicht in ihrer Komplexität beschrieben werden. Hinzu kommt, dass an Ganztagsschulen aktuell ein Potpourri an Koordinationsmodellen zu finden ist, das sich durch unterschiedliche Zeitbudgets, Vergütungen und Weisungsbefugnisse auszeichnet (vgl. Kahnert et al. 2015). Eine mögliche Schlussfolgerung wäre, Regulierungen hinsichtlich des Anstellungsumfangs von Ganztagskoordinator/innen zu formulieren und somit zumindest einen vergleichbaren Minimalkonsens an Ganztagsschulen zu schaffen. Dies könnte einen Beitrag dazu leisten, dass sich die Erwartungen und Forderungen an eine multiprofessionelle Kooperation an Ganztagsschulen nicht in einer „Sphäre des Unbestimmten“ (Fend 2000, S. 61) verlieren und damit örtlichen Aushandlungsprozessen überlassen werden.

\section{Ausblick}

Durch den vorliegenden Beitrag wird ein scheinbar selbstverständliches Forschungsfeld ,wiederbelebt'. Denn nicht nur die Ganztagsschul-, sondern speziell auch die Kooperationsforschung werden durch die Ergebnisse um eine Ermöglichungsbedingung der multiprofessionellen Kooperation an Ganztagsschulen bereichert (Idel et al. 
2019). Die durch den Beitrag initiierten Irritationen und Überlegungen gilt es in weiterführenden Forschungsarbeiten aufzugreifen, um Transparenz darüber zu schaffen, was die multiprofessionelle Kooperation von Ganztagskoordinator/innen erfordert bzw. zu welchen Konsequenzen sie führt. Denn wie bereits einleitend aufgeführt, lässt sich noch immer konstatieren, dass unklar ist, was Ganztagskoordinator/innen an Ganztagsschulen eigentlich machen. Insofern erscheint es auch unklar, inwiefern durch diese sowohl die erhofften Entlastungsbestrebungen für Schulleitungen möglich erscheinen. Um diese Forschungslücke systematisch zu schließen, drängen sich einerseits Fragen auf, die sich auf die Rahmenbedingungen des Standorts konzentrieren (die Tätigkeit der Ganztagskoordination in unterschiedlichen inter-institutionellen Kooperationssettings), andererseits aber auch die personelle Zusammensetzung der Ganztagskoordination (Kooperation mit einem Koordinationsgremium oder mit einer Steuergruppe) fokussieren.

Schließlich sei auf einige Limitationen der Studie hingewiesen. Es wurde bewusst auf eine Gegenüberstellung von Ganztagskoordinationen zweier Schulen verzichtet, um den explorativen Charakter der Fallstudie detailreich abbilden zu können. Die Erklärungskraft der Studie beschränkt sich demnach auf einen Fall, weshalb von einer Generalisierung der Ergebnisse abgesehen werden sollte. Zudem wurde mit der qualitativen Inhaltsanalyse ein Auswertungsverfahren gewählt, das sich auf die manifesten Inhalte des Datenmaterials konzentriert. Eine Interpretation der Inhalte konnte nur durch die mehrjährige Erfahrung an der Schule und damit einhergehend nur durch das Schulporträt vorgenommen werden. Die herausgearbeitete ,Betonung des Neuen' für die multiprofessionelle Kooperation an Ganztagsschulen sollte daher einer kritischen Überprüfung unterzogen werden.

Funding Open Access funding provided by Projekt DEAL.

Open Access Dieser Artikel wird unter der Creative Commons Namensnennung 4.0 International Lizenz veröffentlicht, welche die Nutzung, Vervielfältigung, Bearbeitung, Verbreitung und Wiedergabe in jeglichem Medium und Format erlaubt, sofern Sie den/die ursprünglichen Autor(en) und die Quelle ordnungsgemäß nennen, einen Link zur Creative Commons Lizenz beifügen und angeben, ob Änderungen vorgenommen wurden.

Die in diesem Artikel enthaltenen Bilder und sonstiges Drittmaterial unterliegen ebenfalls der genannten Creative Commons Lizenz, sofern sich aus der Abbildungslegende nichts anderes ergibt. Sofern das betreffende Material nicht unter der genannten Creative Commons Lizenz steht und die betreffende Handlung nicht nach gesetzlichen Vorschriften erlaubt ist, ist für die oben aufgeführten Weiterverwendungen des Materials die Einwilligung des jeweiligen Rechteinhabers einzuholen.

Weitere Details zur Lizenz entnehmen Sie bitte der Lizenzinformation auf http://creativecommons.org/ licenses/by/4.0/deed.de.

\section{Literatur}

Akkerman, S.F., \& Bakker, A. (2011). Boundary crossing and boundary objects. Review of Educational Research, 81(2), 132-169.

Allen, D. (2000). Doing occupational demarcation. The "boundary-work" of nurse managers in a district general hospital. Journal of Contemporary Ethnography, 29(3), 326-356.

Altermann, A., Lange, M., Menke, S., Rosendahl, J., Steinhauer, R., \& Weischenberg, J. (2018). Bildungsbericht Ganztagsschule NRW 
Arnoldt, B., Furthmüller, P., Kielblock, S., \& Gaiser, J.M. (2018). Aktuelle Entwicklungen der ganztagsschulischen Angebote in Deutschland. In M. Schüpbach, L. Frei \& W. Nieuwenboom (Hrsg.), Tagesschulen: Ein Überblick (S. 249-267). Wiesbaden: Springer VS.

Bauer, P. (2014). Kooperation als Herausforderung in multiprofessionellen Handlungsfeldern. In S. Faas \& M. Zipperle (Hrsg.), Sozialer Wandel. Herausforderungen für Kulturelle Bildung und Soziale Arbeit (S. 273-286). Wiesbaden: Springer VS.

Bertelsmann Stiftung (2017). Mehr Schule wagen. Empfehlungen für guten Ganztag. https://www. bertelsmann-stiftung.de/fileadmin/files/Projekte/27_In_Vielfalt_besser_lernen/170511e_ ganztagsschule_doppelseiten_small-mehr-schule-wagen-empfehlung_fuer-guten-ganztag.pdf. Zugegriffen: 19.06.2019

de Boer, H., \& Spies, A. (2014). Kooperationssettings im Kontext inklusiver Grundschulentwicklungsprozesse. In M. Lichtblau, D. Blömer, A.-K. Jüttner, K. Koch, M. Krüger \& R. Werning (Hrsg.), Gemeinsam anders lehren und lernen (S. 186-198). Bad Heilbrunn: Klinkhardt.

Breuer, A., \& Steinwand, J. (2015). Die Reflexion pädagogischer Praxis in Team- und Gruppensitzungen an Ganztagsschulen. In S. Reh, B. Fritzsche, T.-S. Idel \& K. Rabenstein (Hrsg.), Lernkulturen. Rekonstruktion pädagogischer Praktiken an Ganztagsschulen (S. 264-282). Wiesbaden: Springer VS.

Buchna, J., Coelen, T., Dollinger, B., \& Rother, P. (2016). Normalisierte Hierarchie in Ganztagsgrundschulen: Empirische Befunde zur innerorganisationalen Zusammenarbeit von Lehrkräften und weiterem pädagogisch tätigem Personal. Zeitschrift für Soziologie der Erziehung und Sozialisation, 36(3), 281-297.

Chiapparini, E., Kappler, C., Schuler, P., \& Selmani, K. (2018). „Die wissen gar nicht, was wir alles machen“: Befunde zu multiprofessioneller Kooperation im Zuge der Einführung von Tagesschulen in der Stadt Zürich. In E. Chiapparini, R. Stohler \& E. Bussmann (Hrsg.), Soziale Arbeit im Kontext Schule (S. 48-60). Opladen: Barbara Budrich.

Coelen, T., \& Rother, P. (2014). Weiteres pädagogisch tätiges Personal an Ganztagsschulen. In T. Coelen \& L. Stecher (Hrsg.), Die Ganztagsschule. Eine Einführung (S. 111-126). Weinheim: Beltz Juventa.

Dollinger, S. (2014). Ganztagsschule neu gestalten. Bausteine für die Schulpraxis. Weinheim: Beltz.

Fabel-Lamla, M. (2012). Vertrauen in der interprofessionellen Kooperation zwischen Lehrern und Sozialpädagogen. In C. Schilcher, M. Will-Zocholl \& M. Ziegler (Hrsg.), Vertrauen und Kooperation in der Arbeitswelt (S. 195-213). Wiesbaden: Springer VS.

Fend, H. (2000). Qualität und Qualitätssicherung im Bildungswesen. Wohlfahrtsstaatliche Modelle und Marktmodelle. In Qualität und Qualitätssicherung im Bildungsbereich; Schule, Sozialpädagogik, Hochschule. Zeitschrift für Pädagogik, (Bd. 41, S. 55-72). Weinheim: Beltz.

Fischer, N., Klieme, E., Holtappels, H.G., Stecher, L., \& Rauschenbach, T. (2013). Ganztagsschule 2012/2013. Deskriptive Befunde einer bundesweiten Befragung. Frankfurt a.M., Dortmund, Gießen, München: DIPF, IFS, JLU, DJI.

Flick, U., von Kardorff, E., \& Steinke, I. (2017). Was ist qualitative Forschung? Einleitung und Überblick. In U. Flick, E. von Kardorff \& I. Steinke (Hrsg.), Qualitative Forschung. Ein Handbuch (12. Aufl. S. 13-29). Reinbek bei Hamburg: Rowohlt.

Goffman, E. (2016). Wir alle spielen Theater. Die Selbstdarstellung im Alltag (16. Aufl.). München, Berlin: Piper.

Gröhlich, C., Drossel, K., \& Winkelsett, D. (2015). Multiprofessionelle Kooperation in Ganztagsgymnasien. Umsetzung und Rahmenbedingungen. In H. Wendt \& W. Bos (Hrsg.), Auf dem Weg zum Ganztagsgymnasium (S. 178-200). Münster: Waxmann.

Idel, T.-S., Lütje-Klose, B., Grüter, S., Mettin, C., \& Meyer, A. (2019). Kooperation und Teamarbeit in der Schule. In P. Cloos, M. Fabel-Lamla, K. Kunze \& B. Lochner (Hrsg.), Pädagogische Teamgespräche. Methodische und theoretische Perspektiven eines neuen Forschungsfeldes (S. 34-52). Weinheim: Beltz Juventa.

Johnson, B., \& Christensen, L.B. (2017). Educational research. Quantitative, qualitative, and mixed approaches (6. Aufl.). London: SAGE.

Kahnert, J., Hoeft, M., Neuber, K., Lorenz, R., Gerick, J., Jarsinski, S., et al. (2015). Einführung des gebundenen Ganztags an Gymnasien. Praxistipps für die Ganztagskoordination. Münster: Waxmann.

Kielblock, S., \& Gaiser, J. M. (2017). Professionenvielfalt an Ganztagsschulen. In S. Maschke, G. SchulzGade \& L. Stecher (Hrsg.), Jahrbuch Ganztagsschule 2017. Junge Geflüchtete in der Ganztagsschule (S. 113-123). Schwalbach a.T.: Debus Pädagogik.

KMK (2019). Allgemeinbildende Schulen in Ganztagsform in den Ländern in der Bundesrepublik Deutschland - Statistik 2013 bis 2017. https://www.kmk.org/fileadmin/Dateien/pdf/Statistik/ Dokumentationen/GTS_2017_Bericht.pdf. Zugegriffen: 03.07.2019 
Lindner, R. (2007). Die Entdeckung der Stadtkultur. Soziologie aus der Erfahrung der Reportage. Frankfurt a.M.: Campus.

Maykus, S. (2009). Kooperation: Mythos oder Mehrwert? Der Nutzen multiprofessioneller Kooperation der Akteure schulbezogener Jugendhilfe. In F. Prüß, S. Kortas \& M. Schöpa (Hrsg.), Die Ganztagsschule: von der Theorie zur Praxis (S. 307-321). Weinheim: Juventa.

Mayring, P. (2015). Qualitative Inhaltsanalyse. Grundlagen und Techniken. Weinheim: Beltz.

Olk, T., Speck, K., \& Stimpel, T. (2011). Professionelle Kooperation unterschiedlicher Berufskulturen an Ganztagsschulen - Zentrale Befunde eines qualitativen Forschungsprojektes. Zeitschrift für Erziehungswissenschaft, 14, 63-80. Sonderheft 15.

Rahm, S., Rabenstein, K., \& Nerowski, C. (2015). Basiswissen Ganztagsschule. Konzepte, Erwartungen, Perspektiven. Weinheim: Beltz.

Reh, S., \& Breuer, A. (2012). Positionierungen in interprofessionellen Teams - Kooperationspraktiken an Ganztagsschulen. In S.G. Huber \& F. Ahlgrimm (Hrsg.), Kooperation. Aktuelle Forschung zur Kooperation in und zwischen Schulen sowie mit anderen Partnern (S. 185-197). Münster: Waxmann.

Rehm, I. (2018). Von der Halbtags- zur Ganztagsschule. Lehrerprofessionalisierung im Übergang. Wiesbaden: Springer VS.

Sendzik, N., Otto, J., Berkemeyer, N., \& Bos, W. (2012). Das Regionale Bildungsbüro als Boundary-Spanner? Eine Betrachtung des kommunalen Managements interschulischer Netzwerke. In S. Hornberg \& M. Parreira do Amaral (Hrsg.), Deregulierung im Bildungswesen (S. 331-350). Münster: Waxmann.

Silkenbeumer, M., Kunze, K., \& Bartmann, S. (2018). Teil- und zugleich Allzuständigkeit? Rekonstruktionen zu Zuständigkeitsfigurationen und Positionierungen pädagogischer Berufsgruppen in der Organisation Schule. In L. Neuhaus \& O. Käch (Hrsg.), Bedingte Professionalität. Professionelles Handeln im Kontext von Institution und Organisation (S. 130-157). Weinheim: Beltz Juventa.

Speck, K., Olk, T., \& Stimpel, T. (2011). Auf dem Weg zu multiprofessionellen Organisationen? Die Kooperation von Sozialpädagogen und Lehrkräften im schulischen Ganztag. Zeitschrift für Pädagogik, Beiheft, 57, 184-201.

StEG-Konsortium (2017). Studie zur Entwicklung von Ganztagsschulen (StEG). Erhebungen zum Systemmonitoring von 2012 und 2015 (Datensatz). Berlin: IQB.

StEG-Konsortium (2019). Ganztagsschule 2017/2018. Deskriptive Befunde einer bundesweiten Befragung. Frankfurt a.M.: StEG Konsortium.

Steiner, C., \& Tillmann, K. (2011). Koordinierte Vielfalt? Über die Arbeit in multiprofessionellen Ganztagsteams. In K. Speck, T. Olk, O. Böhm-Kasper, H.-J. Stolz \& C. Wiezorek (Hrsg.), Ganztagsschulische Kooperation und Professionsentwicklung (S. 48-68). Weinheim: Beltz Juventa.

Tillmann, K., \& Rollett, W. (2011). Multiprofessionelle Kooperation und Partizipation an Ganztagsschulen. Welche Auswirkung hat die strukturelle Einbindung des weiteren pädagogisch tätigen Personals auf die berufsgruppenübergreifende Zusammenarbeit? In K. Speck, T. Olk, O. Böhm-Kasper, H.-J. Stolz \& C. Wiezorek (Hrsg.), Ganztagsschulische Kooperation und Professionsentwicklung (S. 29-47). Weinheim: Beltz Juventa.

Weidenhaus, G. (2015). Soziale Raumzeit. Berlin: Suhrkamp. 